\title{
Le peuplement paléolithique et mésolithique de la Suisse: la question de l'utilisation des étages montagnards dans les Alpes
}

\section{Pierre Crotti, Lausanne}

\section{Introduction}

L'histoire du peuplement préhistorique du territoire actuel de la Suisse, à l'instar de toutes les régions alpines, est intimement liée aux changements climatiques, à l'origine des crues et décrues glaciaires qui se sont succédé régulièrement durant tout le Pléistocène. Chacun sait que, pendant les épisodes glaciaires proprement dits, les glaciers interdirent toute présence humaine sur la majorité du territoire et, qu'en plus, ils effacèrent, grâce à leur formidable puissance érosive, toute trace d'occupation antérieure, faisant pratiquement tabula rasa. En conséquence, pour le préhistorien s’intéressant au monde alpin, le corpus des données à disposition se trouve-t-il sérieusement tronqué: ce n'est qu'à partir du Tardiglaciaire que l'on peut suivre de manière satisfaisante, et plus ou moins continue, l'histoire du peuplement humain. Ne font exception à cette règle que les territoires épargnés par les glaces lors du dernier Pléniglaciaire, dans le nord de la Suisse, où des vestiges bien conservés attribuables au Paléolithique moyen remontent jusqu'à l'Eémien (vers 130000 BP), comme le démontrent les recherches récentes effectuées près d'Alle (JU). De même, au sein de plusieurs grottes d'altitude dans les Alpes et les Préalpes (Säntis, Simmental, Chablais), quelques traces humaines datant du Würm moyen ont échappé à l'érosion glaciaire.

Dans ce bref bilan du peuplement préhistorique de la Suisse, l'accent sera mis sur l'occupation des territoires d'altitude dans les Alpes, par des populations de chasseurs-cueilleurs exclusivement, antérieures au Néolithique qui, rappelons-le, voit l'émergence de l'élevage, de l'agriculture et de la sédentarisation. Nous verrons que ces espaces montagnards ont été parcourus dès la fin du Paléolithique moyen par les dernières populations de Néandertaliens, puis de nouveau, de manière sporadique, durant l'Azilien, au cours de l'Alleröd. A la faveur de l'amélioration climatique de l'Holocène, on assiste à une intensification de la fréquentation des étages alpins, dès le Mésolithique.

\section{Avant le Pléniglaciaire supérieur, entre 130000 et 30000 BP: le Paléolithique moyen}

Dans les régions situées à l'extérieur de la zone d'extension maximale des glaciers, les vestiges attribuables au Paléolithique inférieur (Acheuléen) se limitent à de rares trouvailles isolées, difficilement datables (LE Tensorer 1998). Ce n'est qu'à partir du stade isotopique 5 que le corpus s'étoffe quelque peu avec des séries bien documentées rattachables au Paléolithique moyen (fig. 1) (Adatte, Rentzel \& KüBler 1991; Le Tensorer 1998). Les occupations les plus anciennes remontent à l'Eémien (stade isotopique 5e), comme l'ont démontré de manière exemplaire les recherches pluridisciplinaires menées sur le site d'Alle Noir-Bois (Jura) dans les années 1990 (Aubry et al. 2000).

Dans les régions alpines, plusieurs gisements d'altitude, en grottes ou abris, épargnés par l'érosion du Pléniglaciaire supérieur, indiquent que les Moustériens occupaient certainement tout le territoire, y compris les étages montagnards, à la faveur de conditions climatiques plus tempérées (interstadiaires) durant le Würm moyen entre 45000 et 30000 BP (JÉQUIER 1975 Praz et al. 2000; SCHMId 1958). Les sites sont très pauvres en vestiges archéologiques et pour la plupart mal conservés. On peut imaginer qu'ils servaient de haltes de chasse, utilisées de manière saisonnière, en relation avec des campements résidentiels installés en plaine ou en fond de vallée. Ces occupations s'insèrent parfaitement dans les cultures moustériennes européennes et ne constituent pas un faciès particulier, propre au monde alpin. Leur mobilité territoriale, caractéristique des sociétés de chasseurs-cueilleurs, est bien illustrée par l'origine des matières premières siliceuses qu'ils utilisaient pour la fabrication de leur outillage. On constate une amplitude des déplacements de l'ordre d'une centaine de kilomètres (Affolter 2002).

Ces grottes d'altitude ont livré également d'abondants restes d'ours des cavernes (Ursus spelaeus): elles servaient de tanières et de lieux d'hibernation, sans relation avec les occupations moustériennes, en dépit des images pittoresques de chasse à l'ours propagées à partir du XIX ${ }^{\mathrm{e}}$ siècle.

\section{Durant le Pléniglaciaire supérieur, entre 30000 et 18000 BP: le Paléolithique supérieur}

Si la plupart du territoire était inaccessible durant le Pléniglaciaire supérieur, la carte de l'extension maximale des glaciers montre qu'une partie de la Suisse actuelle était libre de glace (fig. 2). Cependant, pour la plus grande partie du Paléolithique supérieur, qui correspond en Europe à l'arrivée de l'homme moderne 


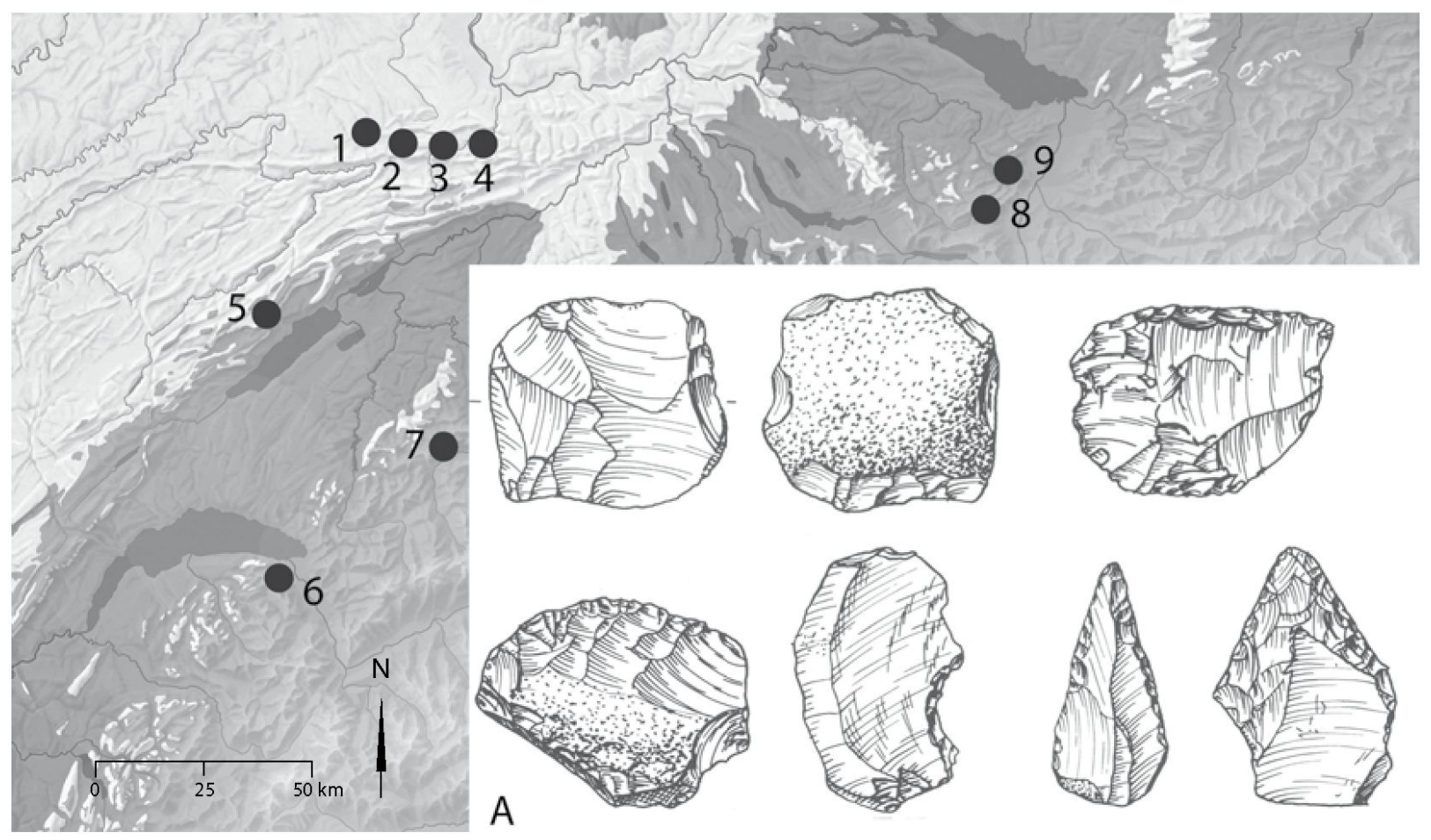

Fig. 1: Suisse: principaux sites du Paléolithique moyen (Moustérien), entre 130000 et 30000 BP, antérieurs au Pléniglaciaire supérieur. En gris foncé, extension maximale des glaciers lors du Pléniglaciaire supérieur (d'après Atlas de la Suisse 2.0).

Jura et nord du Plateau: 1. Noir-Bois et Pré-Monsieur (Alle, JU), 2. Löwenburg (Pleigne, JU), 3. Abri de Liesberg (Liesberg, BE), 4. Kastelhöhle-Nord (Himmelried, SO), 5. Grotte de Cotencher (Rochefort, NE); Alpes et Préalpes: 6. Tanay (Vouvry, VS), alt. $1860 \mathrm{~m}, 7$. Schnuremloch (Oberwil, BE), alt. $1230 \mathrm{~m}, 8$. Wildenmannlisloch (Alt St. Johann, SG), alt. $1637 \mathrm{~m}, 9$. Wildkirchli (Schwende, AI), alt. 1477 m; A. Industrie lithique du Moustérien. Echelle 1:2. (Noir-Bois, d'après Le Tensorer 1998)

Schweiz: Hauptfundorte des mittleren Paläolithikums (Moustérien) zwischen 130000 und 30000 BP, vor dem letzten Pleniglazial. Dunkelgrau gefärbt die maximale Ausdehnung der Gletscher während des letzten Pleniglazials (nach ATLAS DER SCHWEIz 2.0).

Main sites of Middle Palaeolithic period (Moustérien) in Switzerland, dating from 130000 to 30000 BP, before onset of the Würm glacial stage. Maximal glacial advance during the last Pleniglacial stage shaded dark gray (according to AtLas of SwitzerLand 2.0).

(Homo sapiens sapiens), les traces d'occupations humaines font défaut. Les seuls témoignages archéologiques attribuables à cet épisode proviennent du niveau moyen de la grotte de Kastelhöhle-Nord dans le canton de Soleure, daté au radiocarbone vers 19500 BP (Street \& Terberger 2003). Lindustrie lithique se rattache au Badegoulien, une culture qui précède le Madgdalénien, sans qu'une filiation entre les deux ne soit démontrée.

\section{Le Tardiglaciaire, entre 18000 BP et 9700 BCcal: le Magdalénien et l'Azilien}

Après le retrait glaciaire, la flore et la faune vont progressivement coloniser les sols nus abandonnés par les glaciers. Les occupations magdaléniennes, entre 14500 et $12500 \mathrm{BCcal}$, se concentrent sur le Plateau (sites de plein air) et dans le Jura (grottes et abris) (fig. 3) (LEEsch 1993). Il s'agit de populations adaptées à un environnement de steppe froide, un milieu ouvert où les troupeaux de renne et de chevaux constituent le principal gibier. Pour cette période, ce sont les gisements de Champréveyres et de Monruz, au bord du lac de Neuchâtel, fouillés entre 1983 et 1992 à l'occasion des travaux autoroutiers de l'A5, qui constituent les sites de référence (Bullinger, Leesch \& Plumettaz 2006; Leesch 1997; Leesch, CATTIN \& Müller 2004). L'analyse des provenances des matières premières siliceuses des outillages lithiques indique une forte mobilité territoriale des groupes magdaléniens, suivant un axe nord-est sud-ouest sur le Plateau (Moyen Pays), le long 


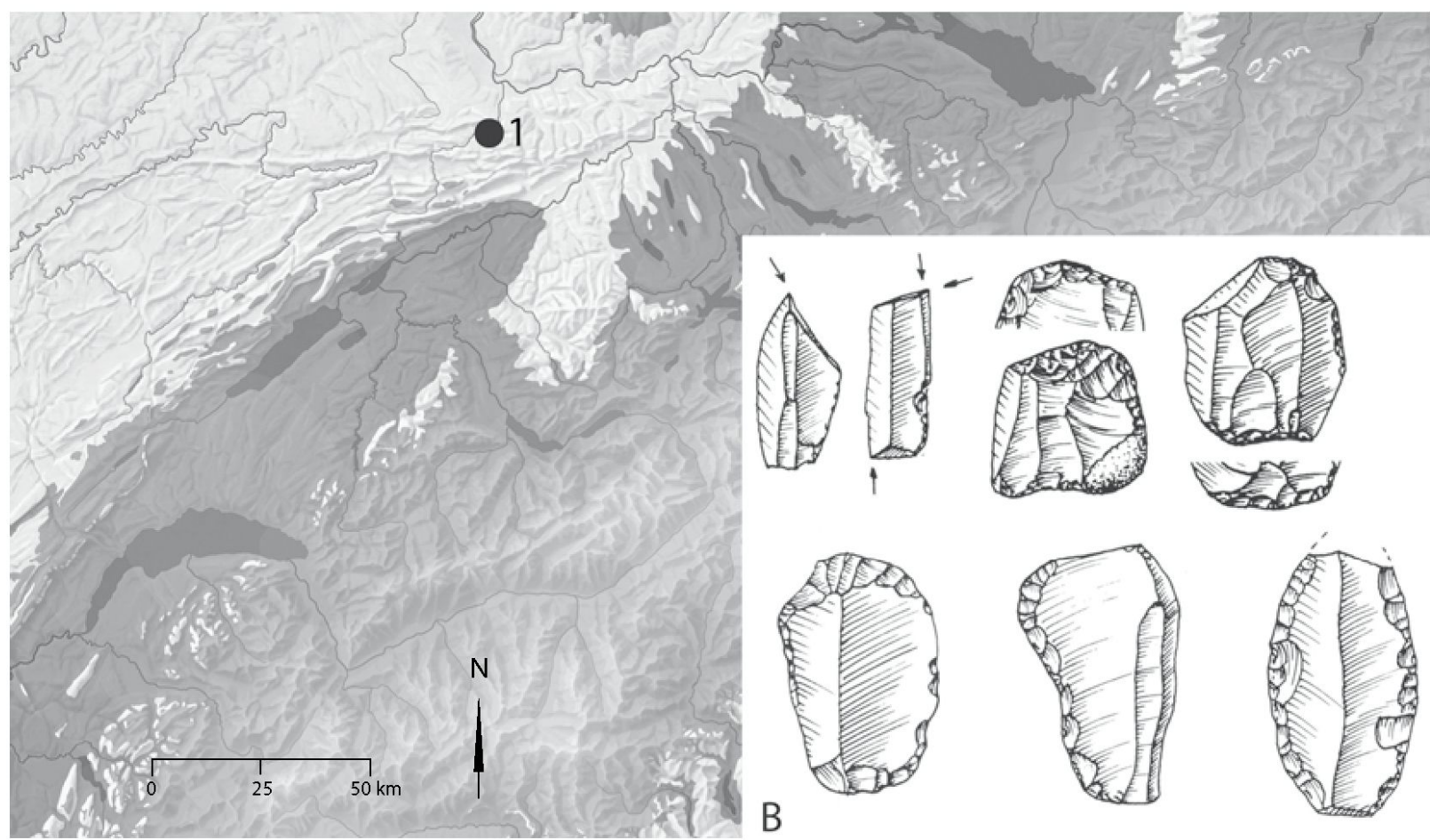

Fig. 2: Suisse: site du Paléolithique supérieur antérieur au Tardiglaciaire, vers 19500 BP. En gris foncé, extension maximale des glaciers lors du Pléniglaciaire supérieur (d'après AtLas de La Suisse 2.0).

1. Kastelhöhle-Nord (Himmelried, SO); B. Industrie lithique du Badegoulien. Echelle 1:2. (Kastelhöhle-Nord, d'après LE TENSORER 1998)

Schweiz: Fundort des Jungpaläolithikums vor dem Spätglazial, um 19500 BP. Dunkelgrau gefärbt die maximale Ausdehnung der Gletscher während des letzten Pleniglazials (nach ATLas DER SCHweIz 2.0).

Upper Palaeolithic site in Switzerland before onset of Late Glacial, around 19500 BP. Maximal glacial advance during the last Pleniglacial shaded dark grey (according to ATLAS OF SWITZERLAND 2.0).

du Jura. Aucun indice ne soutient l'hypothèse d'une utilisation des secteurs d'altitude dans les Alpes pendant le Magdalénien. On ne connaît que des sites «périalpins» à des altitudes ne dépassant pas $900 \mathrm{~m}$, relativement tardifs, attribuables au Magdalénien final: des campement établis en bordure de lacs, comme à Lussy (Châtel-Saint-Denis, FR) (Boisaubert, Pilloud \& MauviLly 1999) et à Einsiedeln-Landgrüti (SZ) (LeUZINGER-PICCAND 1996). De même, les secteurs d'altitude du massif jurassien ne semblent pas avoir intéressé les populations magdaléniennes. Seule une datation sur os de renne provenant de l'abri du Mollendruz (BRIDAULT et al. 2000; Pignat \& Winiger 1998), à près de $1100 \mathrm{~m}$ d'altitude, associée à quelques artefacts lithiques peu caractéristiques, pourrait attester d'une fréquentation de ces étages montagnards à cette époque.

Il faut attendre l'Azilien, qui se développe à partir de $12500 \mathrm{BCcal}$ environ, pour que les premières traces d'occupation d'altitude dans les Alpes soient attestées (fig. 4). A la base de la séquence stratigraphique de l'abri sous bloc de Château-d'Oex (VD) dans les Préalpes occidentales, une pointe azilienne typique indique une présence humaine précoce en milieu montagnard, probablement durant l'Alleröd, entre 11700 et 10900 BCcal. De même, un fragment de pointe azilienne, bien que trouvé hors contexte stratigraphique, provenant du Riedli Balm (Zweisimmen, BE) dans le Simmental, confirme l'utilisation des secteurs d'altitude par les groupes aziliens qui, contrairement à leurs prédécesseurs magdaléniens, se déplacent dans un environnement plus forestier, composé essentiellement de bouleaux et de pins. Les espèces animales de milieu ouvert (cheval, renne), sur lesquelles se basait une grande partie de l'économie de subsistance durant le Magdalénien, ont fait place à un autre gibier (cerf, sanglier), adapté aux nouvelles conditions environnementales.

Durant la péjoration climatique qui marque le Dryas récent, entre 10900 et $9700 \mathrm{BCcal}$, on constate une régression dans le peuplement qui s'accompagne très 


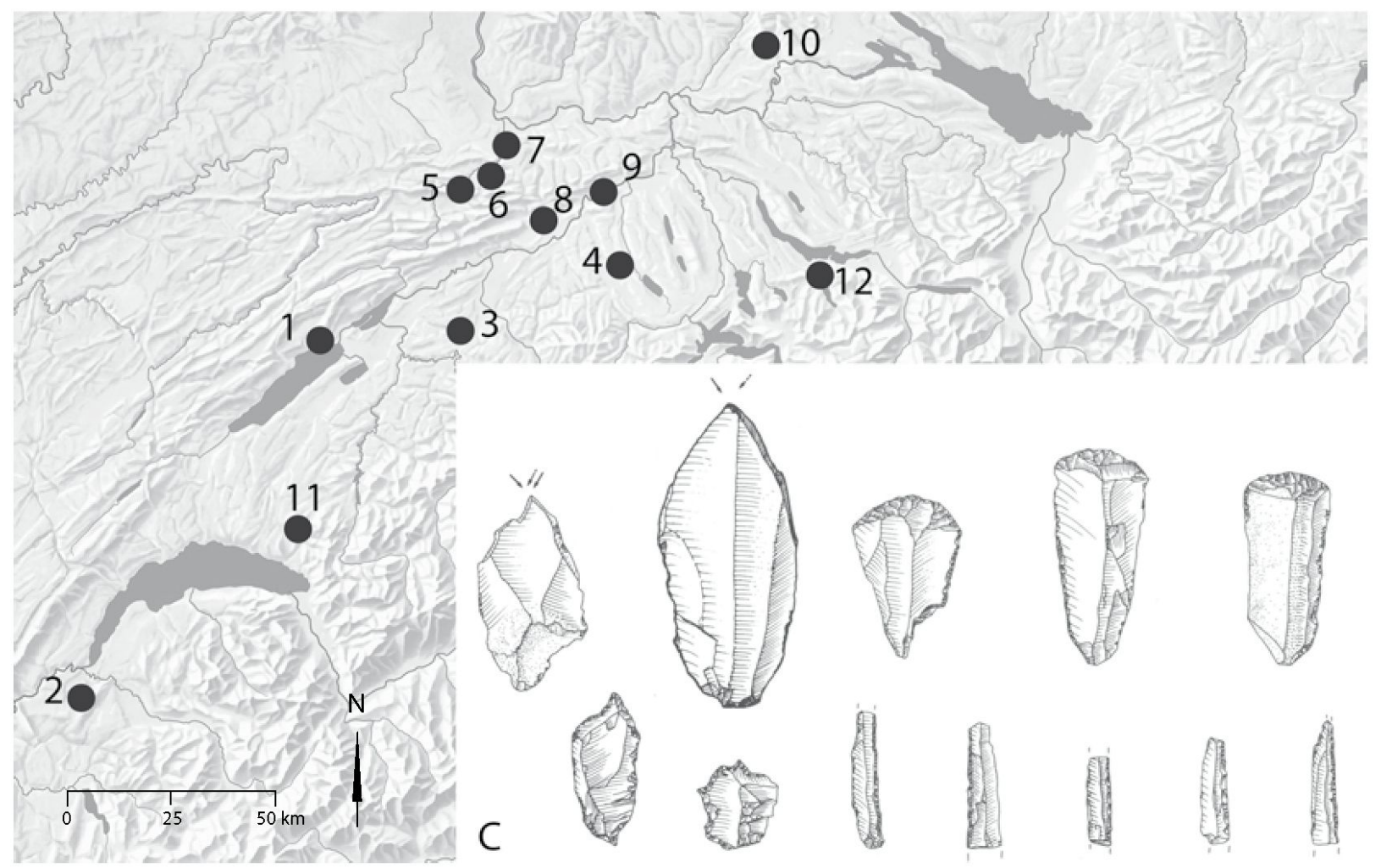

Fig. 3: Suisse: principaux sites du Magdalénien, entre 14500 et 12500 BCcal. (d'après AtLAs de LA SuIsse 2.0) Jura et Plateau: 1. Champréveyres et Monruz (Hauterive et Neuchâtel, NE), 2. Veyrier (Etrembières, Haute-Savoie, France), 3. Moosbühl (Moosseedorf,BE), 4. Reiden Stumpen (Wikon,LU), 5.Kohlerhöhle (Brislach, BE), 6. Hollenberg-Höhle (Arlesheim, BL), 7. Kastelhöhle-Nord (Himmelried, SO), 8. Rislisberghöhle (Oensingen, SO), 9. Köpfli (Winznau, SO), 10. Kesslerloch et Schweizersbild (Thayngen, SH); Préalpes: 11. Lac de Lussy (Châtel-St-Denis, FR), alt. 830 m, 12. Landgrüti Sihlsee (Einsiedeln, SZ), alt. 899 m; C. Industrie lithique du Magdalénien supérieur. Echelle 1:2. (Champréveyres, d'après LeEsch 1993) Schweiz: Hauptfundorte des Magdalénien, zwischen 14500 und 12500 BCcal (nach ATLAS DER SCHWEIz 2.0) Main sites in Switzerland of the Madgalenian dating from 14500 to 12500 BCcal (according to ATLAS OF SWITZERLAND 2.0)

certainement d'un abandon temporaire des étages montagnards. Une occupation de fond de vallée à Coire Marsöl (Nielsen 2003), que le fouilleur place dans le Dryas récent, semble faire exception.

\section{L'Holocène ancien, entre 9700 et 5500 BCcal: l'Epipaléolithique récent et le Mésolithique}

Partout dans les Alpes, on assiste dès le Postglaciaire, avec le développement des cultures mésolithiques, à une multiplication du nombre de sites en altitude, comme le montrent les recherches très actives effectuées dans plusieurs régions de l'arc alpin. En effet, depuis les premières investigations de $\mathrm{B}$. BAGOLINI dans le Trentin dans les années 1970 (BAGolin 1972), les travaux se sont intensifiés, non seulement en Italie septentrionale (Broglio 1992) mais également en France, dans la Chartreuse et le Vercors (BINTZ 1999), et dans le Tyrol autrichien (SCHÄFER 1998), pour ne citer que quelques exemples. La Suisse ne semble pas devoir faire exception, même si les données à disposition restent encore très lacunaires (CROTTI \& Pignat 1992; Crotti 1993, 2002; Crotti et al. 2002a, 2002b). Dernièrement, quelques nouvelles découvertes ont enrichi le corpus, en premier lieu dans les Préalpes occidentales, comme nous le verrons plus loin, mais également dans les Alpes centrales, où plusieurs sites de surface ont été mis en évidence à proximité du Col du Simplon (VS), entre 2000 et $2030 \mathrm{~m}$ d'altitude (Crotti, Curdy \& LeUZINGER 2004). Quelques trouvailles isolées ont également été recensées dans les Grisons, à Lai da Rims (Müstair, 2145 m) (RAGETH 2007) ou en Suisse centrale, à Planggenstafel (Engelberg, OW, 2040 m) (Fricker \& LeUZinger 2006).

\subsection{Les Préalpes de Suisse occidentale}

Notre propos n'est pas ici de discuter l'ensemble des 


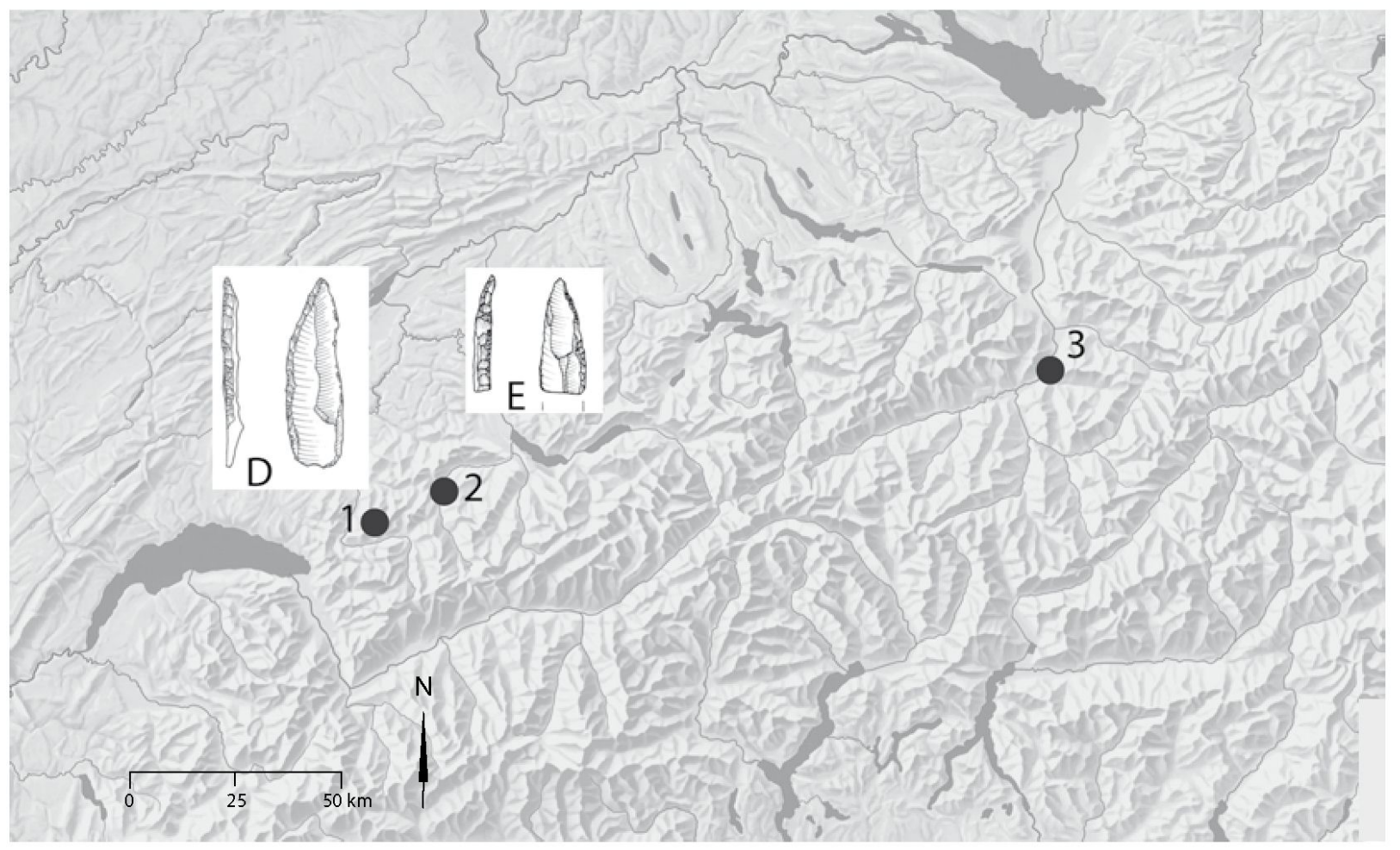

Fig. 4: Sites de l'Azilien dans les Alpes suisses, entre 12000 et 10000 BCcal. (d'après AtLas de La Suisse 2.0) 1. Sciernes-Picats (Château-d'CEx, VD), alt. 1180 m, 2. Riedli Balm (Zweisimmen, BE), alt. 950 m, 3. Marsöl (Chur, GR), alt. 600 m; D. Pointe azilienne. Echelle 1:2. (Sciernes-Picats, dessin B. Nıón), E. Pointe azilienne. Echelle 1:2. (Riedli Balm, dessin B. Nión)

Fundorte des Azilien in den Schweizer Alpen, zwischen 12000 und 10000 BCcal (nach AtLAS DER SCHWEIz 2.0) Main sites in the Swiss Alps of the Azilian dating from 12000 to 10000 BCcal (according to ATLAS OF SWITZERLAND 2.0)

données disponibles pour les Alpes suisses, mais de mettre l'accent sur un cas précis: les Préalpes de Suisse occidentale (fig. 5). Bien qu'encore limités, les résultats à disposition permettent d'esquisser les grandes lignes de l'utilisation des secteurs montagnards par les dernières populations de chasseurs-cueilleurs, avant le changement économique majeur que représente le Néolithique, dès la seconde moitié du $\mathrm{VI}^{\mathrm{e}}$ millénaire avant notre ère.

\subsubsection{L'abri sous bloc de Château-d'Oex (VD)}

Délaissée depuis les premiers travaux entrepris dans le Simmental entre 1925 et 1951 (ANDRIST, FLüKIGER \& ANDRIST 1964), la recherche sur les chasseurscueilleurs préhistoriques dans les Préalpes de Suisse occidentale fut réactivée en 1989 par la découverte de l'abri sous bloc de Château-d'Oex. Implanté à l'altitude de $1200 \mathrm{~m}$ environ, dans une vallée latérale sur la rive droite de la Sarine, le site bénéficie de la proximité immédiate de sources de matières premières siliceuses. L'état de conservation des vestiges est excellent et les restes osseux particulièrement bien préservés. La séquence stratigraphique de l'abri montre une succession d'occupations humaines, dont la plus ancienne, comme nous l'avons vu plus haut, remonte probablement à l'Alleröd. Durant l'Holocène, les occupations s'étagent entre le début du Préboréal, vers $9500 \mathrm{BCcal}$, et l'Atlantique ancien, vers $6000 \mathrm{BCcal}$, et concernent ainsi l'Epipaléolithique récent, le Mésolithique ancien/ moyen et le Mésolithique récent.

L'analyse préliminaire des restes fauniques montre un large éventail d'espèces chassées (cerf, bouquetin, chamois, sanglier, blaireau, renard, lièvre) dénotant une exploitation diversifiée des ressources animales. Aucun témoignage d'une chasse spécifique au milieu montagnard n'est décelable. Les quelques indices de saisonnalité disponibles permettent de proposer des occupations qui s'insèrent entre la mi-juillet et la minovembre, peut-être même entre avril et décembre.

La composition de l'outillage récolté dans l'abri suggère un spectre d'activités assez variées, qui ne paraissent pas liées exclusivement à la chasse. L'ana- 


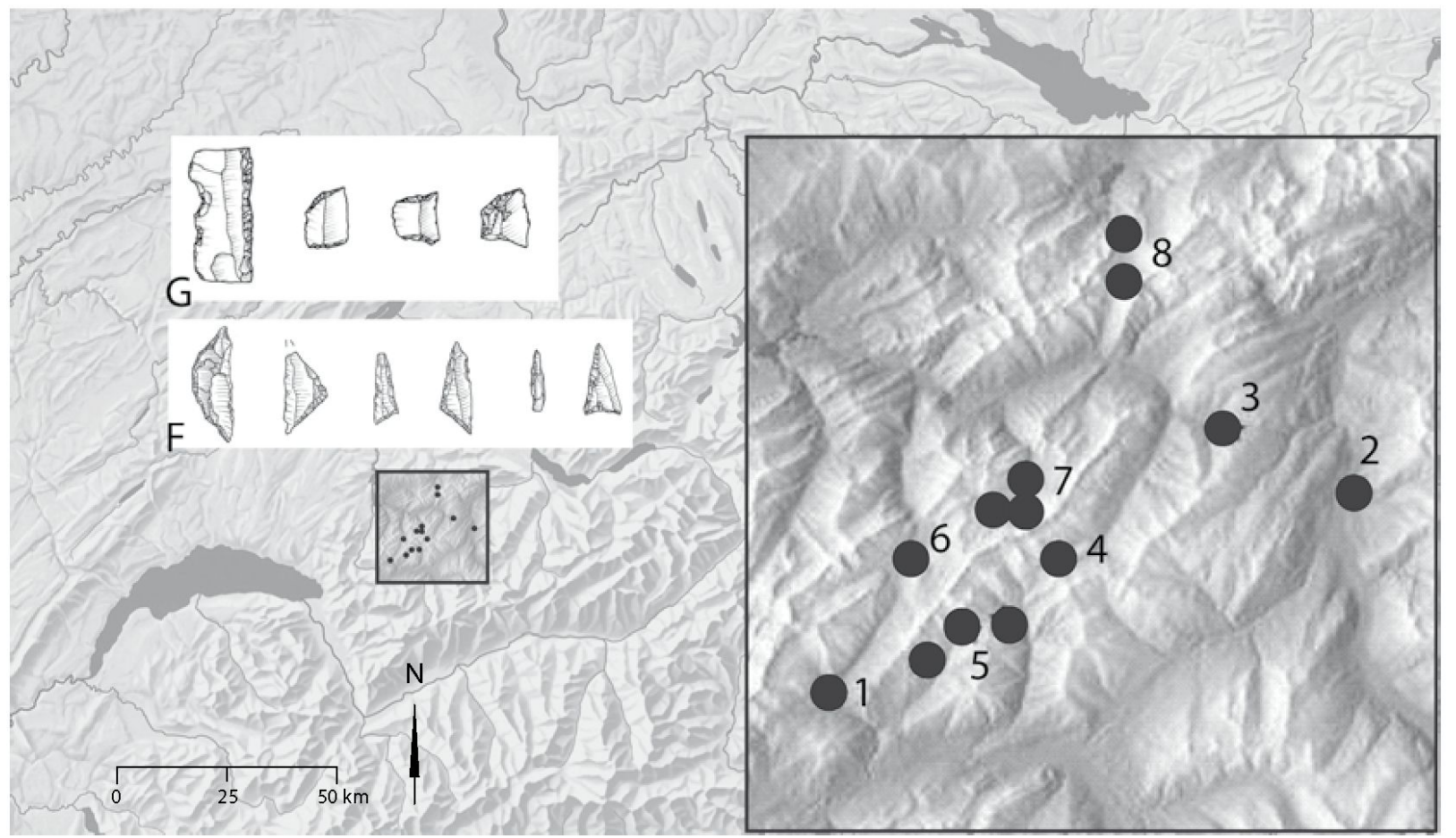

Fig. 5: Sites du Mésolithique dans les Préalpes de Suisse occidentale. Carte simplifiée: les points représentent souvent plusieurs sites, proches les uns des autres (d'après Atlas de la Suisse 2.0).

1. Sciernes-Picats (Château-d'Ex, VD), alt. 1180 m, 2. Riedli Balm (Zweisimmen, BE), alt. 950 m, 3. Jaunpass (Boltigen, BE), alt. 1 500-1 510 m, 4. Mittelberg (Saanen, BE), alt. 1600 m, 5. Les Roseys-La Forcla (Rougemont, VD), alt. 1 420-1 660 m, 6. Le Gros-Mont (Charmey, FR), alt. 1 400-1 700 m, 7. Le Petit-Mont (Charmey, FR), alt. 1 560-1 710 m, 8. Euschelstal (Jaun, FR), alt. 1 350-1 550 m; F. Industrie lithique du Mésolithique ancien/moyen. Echelle 1:2. (Sciernes-Picats, dessin B. Nión), G. Industrie lithique du Mésolithique récent. Echelle 1:2. (Riedli Balm, dessin B. Nión)

Fundorte des Mesolithikums in den Westschweizer Voralpen. Vereinfachte Karte: die Punkte zeigen oft mehrere Fundorte an, die nahe beieinander liegen (nach AtLAS DER SCHweIz 2.0).

Sites in the Prealps of western Switzerland of the Mesolithic. Simplified map: the points represent several sites located close to each other (according to AtLas of Switzerland 2.0).

lyse tracéologique effectuée sur les différents niveaux d'occupation confirme cette impression.

Ainsi, il apparaît que l'abri sous bloc jouait probablement le rôle de site résidentiel, caractérisé par une exploitation diversifiée du monde animal et un outillage lithique multifonctionnel: il ne devait pas se distinguer du point de vue économique des établissements de plaine, installés sur le Plateau, si ce n'est par son utilisation saisonnière, excluant a priori les mois d'hiver.

\subsubsection{Campements de plein air sur le Jaunpass (Boltigen, BE)}

La découverte de l'abri sous bloc de Château-d'Oex constituait une première étape. Dans le monde alpin, plusieurs exemples montrent que les populations mésolithiques exploitaient largement les terroirs à disposition. Par conséquent, il semblait très probable que les occupations mésolithiques dans les Préalpes occidentales ne demeuraient pas confinées dans l'étage montagnard (900-1 $400 \mathrm{~m}$ ); l'existence de campements de plein air installés dans l'étage subalpin inférieur (1 400-1900 m) restait tout de même à démontrer.

Le choix de la première zone à prospecter se porta sur le Jaunpass, dont la configuration correspond bien au modèle observé dans d'autres régions alpines, avec la présence d'un plan d'eau entouré de petites collines, à proximité d'un col. L'hypothèse se vérifia rapidement: au printemps 2000 , quatre premiers sites furent découverts à proximité immédiate du sommet du col, en bordure du marais de Kilchmoos, à des altitudes comprises entre 1500 et 1510 m (CROTTI \& BulLiNGER 2001). 


\subsection{Intensification des prospections}

Les premières découvertes du Jaunpass suscitèrent un fort engouement pour le Mésolithique préalpin et une intense activité de prospection a vu le jour, en particulier sur la commune de Charmey, dans un vaste secteur de l'étage subalpin inférieur qui s'étend entre l'abri sous bloc de Château-d'Oex et le Jaunpass (BraILlard et al. 2003). De nombreux sites de surface ont été découverts, étagés entre 1400 et $1700 \mathrm{~m}$ d'altitude. En l'absence de datations absolues, l'analyse typologique du matériel lithique permet d'identifier le Mésolithique ancien/moyen et le Mésolithique récent; l'Epipaléolithique récent, contemporain du tout début du Préboréal, n'est pas représenté.

\subsection{Un premier bilan}

L'examen des données disponibles sur le Mésolithique des Préalpes de Suisse occidentale, que nous venons de résumer sommairement, permet de dresser un rapide bilan.

La répartition des sites montre tout d'abord la présence d'établissements en fond de vallée, localisés dans l'étage montagnard (900-1400 m), à l'image de Château-d'Oex: des sites stratifiés en abri, indiquant des occupations répétées. Ensuite, on observe des campements de plein air dans l'étage subalpin inférieur, installés entre 1400 et $1700 \mathrm{~m}$ d'altitude dans des contextes topo-géographiques désormais «classiques» (LANzINGER 1996), en relation avec des cols ou des zones de passages, à proximité de petits lacs ou de zones humides. On rencontre également quelques occupations en abri sous roche ou sous bloc. Pour l'instant, l'étage subalpin supérieur n'a pas livré de témoignages mésolithiques.

Les sites de l'étage montagnard ont certainement joué le rôle de sites résidentiels. La fonction des campements de plein air de l'étage subalpin inférieur reste encore à définir. La question serait de savoir de quelle manière et dans quelle mesure ils se différenciaient des sites résidentiels de l'étage montagnard, en plus d'une utilisation saisonnière probablement réduite.

L'analyse des provenances des matières premières siliceuses retrouvées sur les sites permet de se faire une idée du territoire économique des groupes mésolithiques dans la mesure où l'on admet généralement que ces matériaux étaient récoltés par les chasseurscueilleurs au cours de leur déplacements. Dans le cas qui nous intéresse, il est possible de mettre en évidence une forte mobilité territoriale impliquant une circulation entre le Plateau et les Préalpes, sur des distances de l'ordre de 50 à $100 \mathrm{~km}$ (СRоTTI 2006).

En l'absence de données, pour l'instant, sur les autres secteurs du versant nord des Alpes, jouxtant le centre et l'est du Plateau suisse, il n'est pas possible de savoir si l'utilisation des étages montagnard et subalpins par les populations mésolithiques, impliquant des déplacements de l'ordre d'une centaine de kilomètres, était généralisée et homogène; ou si, au contraire, elle était ciblée sur certaines zones présentant un intérêt économique plus marqué comme, par exemple, un accès privilégié aux sources de matières premières siliceuses, à l'image des Préalpes occidentales.

\subsection{Perspectives}

Après ce bref état de la question centré sur une région particulière, choisie en fonction de l'actualité de la recherche, il semble évident que la mise sur pied de travaux de terrain sur une plus large échelle, au niveau national, permettrait d'affiner et de nuancer le tableau général présenté ici. Ceci impliquerait une meilleure connaissance des territoires économiques et des voies de circulation, ainsi qu'une définition plus précise de l'insertion chronologique et de la fonction des différents types de sites.

\section{Conclusions}

Linfluence des facteurs climatiques et environnementaux sur le peuplement humain est évidemment primordiale, en particulier dans les régions alpines qui présentent des conditions géomorphologiques très contrastées. Cependant, en examinant l'histoire des chasseurs-cueilleurs dans les Alpes suisses, on constate que les territoires montagnards ont régulièrement été investis. Dans ce domaine, les importantes lacunes dans nos connaissances relèvent plus de l'état de la recherche que de la réalité historique. Après quatre décennies de fouilles archéologiques concentrées essentiellement autour des centres urbains et des travaux autoroutiers à la faveur d'interventions de sauvetage, le temps est peut-être venu pour les préhistoriens de se tourner vers d'autres axes de recherches et d'investir, à leur tour, les territoires alpins.

\section{Bibliographie}

Adatte, T., Rentzel, P. \& B. Kübler (1991): Étude minéralogique et sédimentologique du remplissage karstique de la grotte de Cotencher (Jura neuchâtelois, Suisse). - In: Eclogae geologicae Helvetiae 84, 3 : 671-688.

Affolter, J. (2002): Provenance des silex préhistoriques du Jura et des régions limitrophes. $-=$ Archéologie neuchâteloise 28, Neuchâtel.

Andrist, D., Flǘkiger, W. \& A. Andrist (1964): Das Simmental zur Steinzeit. - = Acta Bernensia 3, Bern.

Aubry, D., Guélat, M., Detrey, J. \& B. OthennNGIRARD (2000): Dernier cycle glaciaire et occupations paléolithiques à Alle, Noir-Bois. $-=$ Cahiers d'archéologie jurassienne 10 , Porrentruy. 
Bagolin, B. (1972): Primi risultati delle ricerche sugli insediamenti epipaleolitici del Colbricon (Dolomiti). - In: Preistoria alpina 8: 107-149.

Bintz, P. (1999): Peuplements et milieux du Paléolithique final au Mésolithique dans les Alpes du Nord françaises: dynamique et occupations territoriales. - In: Della Casa, P. (éd.): Prehistoric alpine environment, society and economy. - = Universitätsforschungen zur prähistorischen Archäologie 55, Bonn: 101-110.

Boisaubert, J.-L., Pilloud, P. \& M. Mauvilly (1999): Premiers indices d'une occupation magdalénienne en Veveyse. - In: Cahiers d'archéologie fribourgeoise 1, Fribourg: 14-19.

Braillard, L., Menoud, S., Mauvilly, M., Boisaubert, J.-L. \& J.-M. BAERISWYL (2003): Préalpes et chasseurscueilleurs en terres fribourgeoises. - In: Cahiers d'archéologie fribourgeoise 5, Fribourg: 42-69.

Bridault, A., Chaix, L., Pion, G., Oberlin, C., Thiébault, S. \& J. Argant (2000): Position chronologique du renne (Rangifer tarandus L.) à la fin du Tardiglaciaire dans les Alpes du Nord françaises et le Jura méridional. - In: Pıon, G. (éd.): Le Paléolithique supérieur récent: nouvelles données sur le peuplement et l'environnement. - = Mémoire de la Société préhistorique française 28 , Paris: $47-57$

Broglio, A. (1992): Mountain sites in the context of north-east Italian Upper Paleolithic and Mesolithic. - In: Preistoria alpina 28, Trento: 293-310.

Bullinger, J., Leesch, D. \& N. Plumettaz (2006): Le site magdalénien de Monruz. 1: Premiers éléments pour l'analyse d'un habitat de plein air. - = Archéologie neuchâteloise 33, Neuchâtel.

CRoтtr, P. (1993): Spätpaläolithikum und Mesolithikum in der Schweiz: die letzten Jäger. L'Epipaléolithique et le Mésolithique en Suisse: les derniers chasseurs. - In: SPM 1:Die Schweiz vom Paläolithikum bis zum frühen Mittelalter. La Suisse du Paléolithique au Moyen Age, Bâle: 203-243.

СRотті, P. (2002): Mesolithic settlement of the Central Alps and the use of the mountain sectors. - In: Preistoria alpina 34 , Trento: 101-110.

Свотті, P. (2006): Between the Alps and the Jura - Economy and Mobility during the Mesolithic in Western Switzerland. - In: KIND, C.-J. (ed.): After the Ice Age. Settlements, subsistence and social development in the Mesolithic of Central Europe. Proceedings of the international conference, $9^{\text {th }}$ to $12^{\text {th }}$ of September 2003, Rottenburg/Neckar, Baden-Württemberg, Germany. - = Materialhefte zur Archäologie in Baden-Württemberg 78, Stuttgart: 129-134.

Crotti, P. \& J. Bullinger (2001): Campements mésolithiques d'altitude sur le Jaunpass (Simmental, canton de Berne, Suisse). - In: Annuaire de la Société suisse de préhistoire et d'archéologie 84, Bâle: 119-124.

Crotti, P., Curdy, P. \& U. Leuzinger (2004): La région du Simplon (Valais), du Mésolithique à l'époque moderne. Campagne de prospection 2003. - In:
Annuaire de la Société suisse de préhistoire 87, Bâle: 271-278.

Crotti, P. \& G. Pignat (1992): L'utilisation des étages montagnards durant le Mésolithique dans les Alpes suisses. - In: Preistoria alpina 28, Trento: 275-284.

Crotti, P., Pignat, G. \& A.-M. Rachoud-Schneider (dir.) (2002a): Premiers hommes dans les Alpes: de 50000 à 5000 avant Jésus-Christ. - Lausanne: Payot, Sion: Musées cantonaux du Valais.

Crotti, P., Pignat, G. \& A.-M. Rachoud-Schneider (dir.) (2002b): Die ersten Menschen im Alpenraum von 50000 bis 5000 vor Christus. - Zürich:Verlag Neue Zürcher Zeitung, Sitten: Walliser Kantonsmuseen.

Fricker, H.-R. \& U. LeUzinger (2006): Engelberg, OW, Planggenstafel, 2040 m. - In: Annuaire d'archéologie Suisse 89, Bâle: 217-218.

JÉQUIER, J.-P. (1975): Le moustérien alpin, révision critique. $-=$ Eburodunum 2, Yverdon .

LANZINGER, M. (1996): Sistemi di insediamento mesolitici come adattamento agli ambienti montani. - In: Kozlowski, S.K. \& C. Tozzi (éds): The Mesolithic. $13^{\text {th }}$ International Congress of Prehistoric and Protohistoric Sciences, Forlì, Italia, 8-14 September 1996. - Colloquia 7, Forlì: 125-140.

Le Tensorer, J.-M. (1998): Le Paléolithique en Suisse. - Grenoble: J. Millon.

Leesch, D. (1993): Das späte Jungpaläolithikum. Le Paléolithique supérieur récent. - In: SPM 1: Die Schweiz vom Paläolithikum bis zum frühen Mittelalter. La Suisse du Paléolithique au Moyen Age, Bâle: 153-186.

LEESCH, D. (dir.) (1997): Un campement magdalénien au bord du lac de Neuchâtel: cadre chronologique et culturel, mobilier et structures, analyse spatiale (secteur 1). - = Archéologie neuchâteloise 19, Neuchâtel.

Leesch, D., Cattin, M.-I. \& W. Müller (2004):Témoins d'implantations magdaléniennes et aziliennes sur la rive nord du lac de Neuchâtel. $-=$ Archéologie neuchâteloise 31, Neuchâtel.

LeuZinger-Piccand, C. (dir.) (1996): Einsiedeln SZLangrüti: eine spätmagdalénienzeitliche und mesolithische Freilandstation in den Voralpen: Grabungsbericht und Sammlungsstudie. - In: Annuaire de la Société suisse de préhistoire et d'archéologie 79, Bâle: 7-26.

NieLSEN, E. (2003): Chur, Marsöl. Eine spätpaläolithische Fundstelle im Bündner Rheintal. - In: Jahresbericht des Archäologischen Dienstes Graubünden und der Denkmalpflege Graubünden, Chur: 48-72.

Pignat, G. \& A. Winiger (1998): Les occupations mésolithiques de l'abri du Mollendruz: abri Freymond commune de Mont-la-Ville (VD, Suisse). - = Cahiers d'archéologie romande 72, Lausanne.

Praz, J.-C., Curdy, P., Leuzinger, U., Leuzinger-PicCAND, C. \& M. Schweizer (2000): Paléolithique alpin à Taney (commune de Vouvry VS). - In: Annuaire de la Société suisse de préhistoire et d'archéologie 83, Bâle: 25-35. 
Rageth, J. (2007): Müstair, GR, Lai da Rims, 2145 m. - In: Annuaire d'archéologie Suisse 90, Bâle: 138. SCHÄFER, D. (1998): Zum Untersuchungstand auf dem altmesolithischen Fundplatz vom Ullafelsen im Fotschertal (Stubaier Alpen, Tirol). - In: Germania 76: 439-496.

Schмid, E. (1958): Höhlenforschung und Sedimentanalyse: ein Beitrag zur Datierung des Alpinen Paläolithikums. $-=$ Schriften des Instituts für Ur- und Frühgeschichte der Schweiz 13, Basel.

Street, M. \& T. Terberger (2003): New evidence for the chronology of the Aurignacian and the question of pleniglacial settlement in western Central Europe. - In: D'Errico, F. \& J. Zilhao (éds): The chronology of the Aurignacian and of the transitional technocomplexes. Dating, stratigraphies, cultural implications. - = Proceedings of the $14^{\text {th }}$ UISPP (Union internationale des sciences préhistoriques et protohistoriques) Congress at Liège, September 2001, Lisboa: 213-222.

\section{Résumé: Le peuplement paléolithique et mésolithique de la Suisse: la question de l'utilisation des étages mon- tagnards dans les Alpes \\ L'histoire du peuplement préhistorique de la Suisse, à l'image de tous les pays alpins, est évidemment lar- gement tributaire des fluctuations glaciaires. Le bilan proposé dans cet article est axé en priorité sur les occu- pations humaines des territoires d'altitude dans les Alpes, au cours du Paléolithique et du Mésolithique. Ces espaces ont été parcourus dès la fin du Paléolithi- que moyen, entre 45000 et $35000 \mathrm{BP}$. Après le retrait glaciaire, les premières traces humaines dans l'étage montagnard remontent à l'Azilien et sont contempo- raines de l'Alleröd. Avec l'amélioration climatique de l'Holocène, les témoignages d'occupation des terri- toires alpins par des populations du Mésolithique se multiplient, même si les recherches dans ce domaine sont encore peu développées, en comparaison avec d'autres régions des Alpes.}

Mots-clés: Alpes, glaciation, Paléolithique, Mésolithique, étages montagnards

\section{Zusammenfassung: Die paläolithische und mesoli- thische Besiedlung der Schweiz und die Nutzung der Bergstufe in den Alpen}

Die prähistorische Nutzung des Alpenraums ist offensichtlich stark von der Vergletscherung abhängig. Ziel dieses Artikels ist es, die Geschichte der menschlichen Besiedlung insbesondere in der Bergstufe der Schweiz während des Paläolithikums und des Mesolithikums zusammenzufassen. Diese Gebirgsregion wurde bereits seit dem mittleren Paläolithikum, also vor etwa 45000 bis 35000 Jahren (von Menschen) genutzt. Nach dem Rückzug der Gletscher werden die ersten menschlichen Spuren in der Bergstufe in das Azilien datiert, zur Zeit des Alleröds. Im frühen Holozän wird die jahreszeitliche Besiedlung der Bergstufe durch die mesolithische Bevölkerung klar bestätigt, obwohl der Forschungsstand dazu in der Schweiz, im Vergleich mit anderen Alpenländern, noch wenig entwickelt ist.

Schlüsselwörter: Alpen, Vergletscherung, Paläolithikum, Mesolithikum, Bergstufen

\begin{abstract}
Palaeolithic and Mesolithic settlement in Switzerland and use of the mountain zone in the Alps The prehistoric land use of the alpine area is obviously closely related to the glaciations. The aim of this paper is to summarize the history of human occupations in Switzerland during the Palaeolithic and the Mesolithic, focusing on the mountain zone. These territories were already used at end of the Middle Palaeolithic, between 45000 and $35000 \mathrm{BP}$. After deglaciation, the first human traces in the mountain zone are dated to the Azilian culture during the Alleröd. In the Early Holocene, the seasonal occupation of the mountain zone by Mesolithic populations is clearly established, although research in this field remains, for the moment, less developed in Switzerland than in other regions of the Alps.
\end{abstract}

Keywords: Alps, glaciation, Palaeolithic, Mesolithic, mountain zone

Pierre Crotti, Conservateur, Musée cantonal d'archéologie et d'histoire, Palais de Rumine, Place de la Riponne 6, CH-1014 Lausanne, Suisse.

e-mail: pierre.crotti@vd.ch

\section{Manuskripteingang/received/manuscrit entré le} 8.5.2008

Annahme zum Druck/accepted for publication/accepté pour limpression: 9.9.2008 